\title{
OPEN Structural basis for the structural dynamics of human mitochondrial chaperonin $\mathrm{mHsp} 60$
}

\begin{abstract}
Joseph Che-Yen Wang ${ }^{1 \bowtie}$ \& Lingling Chen ${ }^{2 \bowtie}$
Human mitochondrial chaperonin $\mathrm{mHsp} 60$ is essential for mitochondrial function by assisting folding of mitochondrial proteins. Unlike the double-ring bacterial GroEL, $\mathrm{mHsp60}$ exists as a heptameric ring that is unstable and dissociates to subunits. The structural dynamics has been implicated for a unique mechanism of $\mathrm{mHsp60}$. We purified active heptameric $\mathrm{mHsp60}$, and determined a cryo-EM structure of $\mathrm{mHsp} 60$ heptamer at 3.4 $\AA$. Of the three domains, the equatorial domains contribute most to the inter-subunit interactions, which include a four-stranded $\beta$ sheet. Our structural comparison with GroEL shows that $\mathrm{mHsp} 60$ contains several unique sequences that directly decrease the sidechain interactions around the $\beta$ sheet and indirectly shorten $\beta$ strands by disengaging the backbones of the flanking residues from hydrogen bonding in the $\beta$ strand conformation. The decreased inter-subunit interactions result in a small inter-subunit interface in $\mathrm{mHsp} 60$ compared to GroEL, providing a structural basis for the dynamics of $\mathrm{mHsp} 60$ subunit association. Importantly, the unique sequences are conserved among higher eukaryotic mitochondrial chaperonins, suggesting the importance of structural dynamics for eukaryotic chaperonins. Our structural comparison with the single-ring $\mathrm{mHsp} 60-\mathrm{mHsp} 10$ shows that upon $\mathrm{mHsp} 10$ binding the shortened inter-subunit $\beta$ sheet is restored and the overall inter-subunit interface of $\mathrm{mHsp} 60$ increases drastically. Our structural basis for the mHsp10 induced stabilization of $\mathrm{mHsp} 60$ subunit interaction is consistent with the literature that $\mathrm{mHsp} 10$ stabilizes $\mathrm{mHsp} 60$ quaternary structure. Together, our studies provide structural bases for structural dynamics of the $\mathrm{mHsp} 60$ heptamer and for the stabilizing effect of $\mathrm{mHsp} 10$ on $\mathrm{mHsp60}$ subunit association.
\end{abstract}

Human mitochondrial mHsp60 is essential for mitochondria as it is required for mitochondrial protein transport, folding and assembly ${ }^{1-4}$. Due to its roles in human health, $\mathrm{mHsp} 60$ has been proposed as potential biomarkers and drug targets ${ }^{5-8}$. $\mathrm{mHsp} 60$ belongs to an essential protein family called chaperonin that are conserved among the three kingdoms of life ${ }^{9}$. The main function of chaperonins is to mediate folding of cellular proteins. Detailed molecular mechanism of chaperonins has been extensively investigated using the $E$. coli chaperonin GroEL and its cochaperonin GroES ${ }^{10-14}$. GroEL consists of 14 subunits assembled in two heptameric rings forming two central cavities, and each subunit consists of three domains, apical, intermediate and equatorial domains. Proteins in non-native conformations bind to the central cavity, and in an ATP-dependent mechanism they are sequestered into the cavity upon GroES binding to the end of the substrate-loaded cis heptameric ring. The enclosed GroEL-GroES chamber provides the substrate protein an isolated environment favorable for folding. Binding of ATP to a second GroEL ring, trans to the substrate-loaded GroEL-GroES chamber, trigger dissociation of the GroEL-GroES folding chamber, releasing the substrate protein. In support of the essential role of the second ring in GroEL, a single-ring GroEL, GroEL ${ }^{\mathrm{SR}}$, where conserved inter-ring contacts including a conserved salt bridge are disrupted, is nonfunctional with GroES in vivo ${ }^{15}$, because the GroEL ${ }^{\text {SR }}$-GroES complex does not dissociate ${ }^{16}$.

Unlike GroEL, mHsp60 exists as single ring ${ }^{17}$, and $\mathrm{mHsp} 60$ lacks the two pairs of salt bridge found in the inter-ring interface in the double-ring GroEL ${ }^{18}$, suggesting that $\mathrm{mHsp} 60$ and its cochaperonin $\mathrm{mHsp} 10$ may function in a single-ring mechanism other than the double-ring mechanism of GroEL-GroES. In support of the single-ring mechanism, mHsp60 interacts transiently with $\mathrm{mHsp} 10^{19}$, thus the dissociation of the mHsp60mHsp10 chamber does not require the action from a second ring. However, an earlier model ${ }^{20-23}$, supported by the crystal structure of an inactive $\mathrm{mHsp} 60$ variant ${ }^{24}$ complexed with $\mathrm{mHsp} 10$ in a double-ring football-shaped conformation $^{25}$, assumes that $\mathrm{mHsp} 60$ functions via a GroEL-like double-ring mechanism. In the recent revised

\footnotetext{
${ }^{1}$ Department of Microbiology and Immunology, The Pennsylvania State University College of Medicine, 500 University Drive, Hershey, PA 17033, USA. ${ }^{2}$ Department of Molecular and Cellular Biochemistry, 212 S. Hawthorne Dr., Bloomington, IN 47405, USA. ${ }^{\circledR}$ email: cwang6@pennstatehealth.psu.edu; linchen@indiana.edu
} 
model, the single-ring mechanism has been incorporated in addition to the double-ring mechanism ${ }^{26}$. In the studies, cryo-EM shows that $\mathrm{mHsp} 60$ forms both the single- and double-ring mHsp60-mHsp10 complexes in the presence of ADP. Structure-based mHsp60 variants of single-ring only and covalent bonded double-ring are shown active in folding of a substrate protein and in substituting GroEL-GroES to support E. coli cell growth. Thus, it is proposed that mHsp60-mHsp10 can function as either single- or double-ring mechanisms based on the specific mitochondrial substrates.

Detailed mechanistic investigations on $\mathrm{mHsp} 60$-mHsp10 had been hindered by difficulties in preparing mHsp60 until recently. Purified mHsp60 dissociates readily to nonfunctional monomers ${ }^{17,27}$, and mHsp60 has been purified as a double-ring conformation that when in the presence of mHsp10 cannot hydrolyze ATP without the substrate protein ${ }^{21}$, highlighting the difficulties in $\mathrm{mHsp} 60$ preparation. In this report, we purified active $\mathrm{mHsp60}$ in single-ring conformation, and determined mHsp60 structure using cryo-EM. We found that mHsp60 contains several unique residues that decease inter-subunit sidechain interactions and shorten the inter-subunit $\beta$ sheet, resulting in reduced inter-subunit interface when compared with GroEL. These residues are conserved among higher eukaryotic mitochondrial chaperonins. Our structural analysis with the reported single-ring $\mathrm{mHsp60-mHsp10} \mathrm{shows} \mathrm{that} \mathrm{the} \mathrm{mHsp} 60$ subunit interface is increased as a result of $\mathrm{mHsp} 10$ binding. Note that a cryo-EM structure of $\mathrm{mHsp} 60^{28}$ was reported during our manuscript submission, however, without structural analysis on inter-subunit interactions and discussion on their roles on the structural dynamics of mHsp60.

\section{Results}

Purified $\mathrm{mHsp} 60$ is single ring and active. To examine conformation of our purified mHsp60, we used size-exclusion chromatography (SEC) and dynamic light scattering (DLS) to compare mHsp60 with the doublering GroEL and single-ring GroEL ${ }^{\mathrm{SR}}$. As shown in Fig. 1a and Table 1, mHsp60 eluted at the same volume as that of GroEL ${ }^{\mathrm{SR}}$, but later than that of the double-ring GroEL. Also, mHsp60 existed as a particle with a size similar to that of GroEL ${ }^{\mathrm{SR}}$, but smaller than that of GroEL (Fig. $1 \mathrm{~b}$ and Table 1). All three samples were monodisperse since their Polydispersity Index (PDI) were $<0.1$. Thus, our purified $\mathrm{mHsp} 60$ was homogenously in a single-ring conformation, which was further supported by negative stained TEM image (Fig. S1).

We next examined the biochemical activities of the purified $\mathrm{mHsp} 60$. $\mathrm{mHsp} 60$ hydrolyzed ATP at a rate $25 \%$ of GroEL (Table 1), consistent with the previous reports ${ }^{19,20,29}$. The intrinsic ATPase rate of mHsp60 was decreased by $\sim 50 \%$ in the presence of $\mathrm{mHsp} 10$ : the cochaperonin inhibition on the ATPase of the chaperonin is a property found in the chaperonin systems. Moreover, mHsp60-mHsp10 was as effective as GroEL-GroES in mediating folding of malate dehydrogenase (MDH) (Fig. 1c and Table 1). Thus, our purified mHsp60 was biochemically active.

Cryo-EM analysis of $\mathrm{mHsp60.} \mathrm{Cryo-EM} \mathrm{micrographs} \mathrm{also} \mathrm{confirm} \mathrm{the} \mathrm{single-ring} \mathrm{conformation} \mathrm{of}$ mHsp60 (Fig. 2a). Top and bottom views of the $2 \mathrm{D}$ class averages shows seven-fold symmetry characteristics (Fig. 2b), confirming the heptameric arrangement of the mHsp60 subunits. The two stripes in the side view correspond to the apical and equatorial domains, indicating a single-ring conformation. Thus, EM studies validated our purified mHsp60 as a single-ring heptamer. The final cryo-EM map was estimated to 3.4 A (Table 2, Fig. S2) with various local resolutions (Fig. 2c). The map (Fig. 2d) shows that the equatorial domain has the highest resolution $(3.3 \AA$ ) , while the apical domain has the lower resolution range (3.9-4.5 $)$ ). Since high resolution maps reflect a homogenous ensemble of conformational states, our observations indicate that the mHsp60 equatorial domains are structurally more homogenous and better ordered than the apical domains, in agreement with crystal structural analysis of GroEL ${ }^{30,31}$. The homogenous and well-ordered structure of the equatorial domains is attributed by their extensive inter-subunit interactions; they contribute $\sim 75 \%$ of the total inter-subunit interface in the heptamer. The regional map in the equatorial domain that is involved in the inter-subunit interactions (below) is well resolved with clear sidechain information (Fig. 2e).

Overall structure of $\mathrm{mHsp60.} \mathrm{The} \mathrm{mHsp60} \mathrm{heptameric} \mathrm{ring} \mathrm{is} \mathrm{larger} \mathrm{than} \mathrm{a} \mathrm{GroEL} \mathrm{ring.} \mathrm{The} \mathrm{diameter} \mathrm{of}$ the mHsp60 ring is $148.8 \AA, 8 \AA$ wider than that of the GroEL ring (PDB: $5 w 0 s ; 3.5 \AA$ cryoEM structure)-the diameter of the chaperonin ring is determined by the equatorial domains. Since the apical domains contribute to the height of the chaperonin ring, their lower resolution regional map in $\mathrm{mHsp60}$ may not estimate the height of the mHsp60 ring (74.4 $\AA$ ) accurately compared to that of the GroEL ring (69.1 $)$ ); however, mHsp60 appears taller than the GroEL ring (Fig. 3a). A larger mHsp60 ring is also evident by comparing cryo-EM reconstructions (Fig. S3) of mHsp60 and GroEL (EMDB: 8750). The larger size of the mHsp60 ring suggests that the seven $\mathrm{mHsp60}$ subunits may not interact as strongly as the GroEL subunits in the GroEL ring.

Between mHsp60 and GroEL, the three domains, apical, intermediate and equatorial domains, are arranged similarly but differ in their conformations. While conformations of the equatorial domains overlay well, those of the apical domains deviate (Fig. 3b). Overall, mHsp60 consists of shorter $\alpha$ helices and $\beta$ strands than GroEL (Fig. 3c). $292 \mathrm{mHsp} 60$ residues while 392 GroEL residues are involved in forming either $\alpha$ helices or $\beta$ strands (Fig. 3c). The lower secondary structure content of mHsp60 suggests that the mHsp60 subunits are less well folded than the GroEL subunits.

Reduced inter-subunit interactions in $\mathrm{mHsp60.} \mathrm{The} \mathrm{equatorial} \mathrm{domains} \mathrm{contribute} \mathrm{most} \mathrm{of} \mathrm{the} \mathrm{inter-}$ subunit interactions, accounting for $\sim 75 \%$ of the total inter-subunit interface and 44 residues involved in intersubunit interactions. The apical domains contribute nine residues for the inter-subunit interactions, while the intermediate domains contribute six residues. Overall, $\mathrm{mHsp} 60$ has a much smaller inter-subunit interface than GroEL, $\sim 1258 \AA^{2}$ versus $\sim 1674 \AA^{2}$. In the following, we compare the molecular interactions on the inter-subunit 

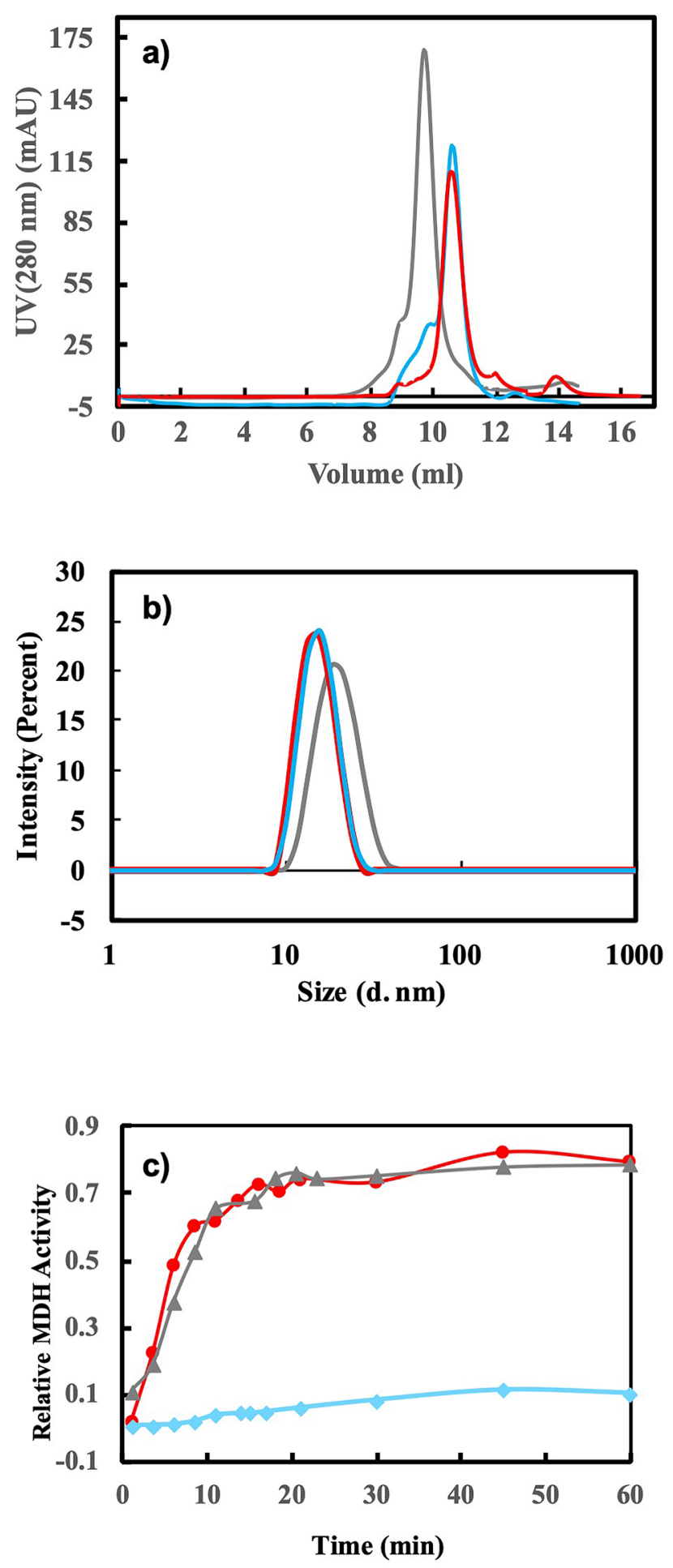

Figure 1. Characterizations of mHsp60. (a) Size exclusion chromatographic profiles, (b) dynamic light scattering analysis, (c) refolding malate dehydrogenase (MDH) of double-ring GroEL (grey), single-ring GroEL $^{\mathrm{SR}}$ (cyan) and mHsp60 (red). The results are summarized in Table 1.

interface between mHsp60 and GroEL. We focus on the equatorial domains because they contribute most of the inter-subunit interactions and their regional maps are well resolved with clear sidechain information.

Center on the inter-subunit interface is a four-stranded $\beta$-sheet: $\beta 1$ and $\beta 18$ from one subunit connected with $\beta 2$ and $\beta 3$ from the other subunit (Fig. 3a). $\beta$ strands are shorter in mHsp60 than in GroEL: 12 mHsp60 residues compared with 19 GroEL residues are involved in the $\beta$-sheet (Table 3). Specifically, $\beta 1$ and $\beta 18$ are shortened to 


\begin{tabular}{|l|l|l|l|l|}
\hline Protein & Elution volume $(\mathbf{m l})^{\mathbf{a}}$ & Z-Average size $(\mathbf{d} . \mathbf{n m})$ & ATPase $\left(\mathbf{m i n}^{-\mathbf{1}}\right)^{\mathbf{c}}$ & MDH yield \\
\hline mHsp60 & 10.62 & $14.85(0.09)^{\mathrm{b}}$ & $\begin{array}{l}0.099 \pm 0.012 \\
0.052 \pm 0.012(\mathrm{w} / \mathrm{mHsp} 10)\end{array}$ & $77.5 \% \pm 2.0 \%$ \\
\hline GroEL & 10.62 & $14.62(0.08)$ & $0.456 \pm 0.020$ & $10.3 \% \pm 2.1 \%$ \\
\hline GroEL & 9.53 & $18.69(0.07)$ & $0.422 \pm 0.020$ & $77.8 \% \pm 1.9 \%$ \\
\hline
\end{tabular}

Table 1. The molecular properties of mHsp60, GroEL ${ }^{\mathrm{SR}}$ and GroEL. a $S E C$ was carried out using a Superdex 200 10/300 GL column (GE Healthcare Life Sciences). ${ }^{\text {b}}$ Values in parentheses refer to Polydispersity Index (PDI). ${ }^{c}$ ATPase activity is normalized to the monomer concentration. ${ }^{\mathrm{d}} \mathrm{MDH}$ yield was at $60 \mathrm{~min}$ after the unfolded MDH was added to the chaperonin systems, and was normalized to native MDH. Without the chaperonin system, the unfolded MDH did not refold $(<5 \%)$.

three residues, the minimal length for $\beta$-strands, from five and seven residues in GroEL, respectively. Together, four hydrogen bonds from the backbones are lost in the reduced-size $\beta$-sheet of mHsp60 (more below).

The shortened $\beta$ strands in mHsp60 are associated with the unique mHsp60 sequences flanking the $\mathrm{N}$ - or C-termini of the $\beta$ strands. At the outer side of the $\beta$-sheet, the GroEL N37 interacts closely with T516 (2.7 $\AA)$ and is proximity with T517 (4.5 $\AA$ ); both T516 and T517 are from the other GroEL subunit (Table 3). These interactions impose restrains on the backbone of N37, leading to a twisted N-terminus of $\beta 2$ at N37 (Fig. 4A). Also, they together with the R36-E518 interaction (next) result in a tight twist N-terminus of $\beta 18$. The GroEL R36 forms a strong charge-charge interaction with E518 (2.8 $)$ and a weak charge-polar interaction with M114 (4.2 A) from the neighboring subunit. These restrains on T517 (via N37) and E518 (via R36) explain the T517-E518 segment tilts $\sim 45^{\circ}$ from the rest of $\beta 18$ (Fig. $4 \mathrm{~A}$ ). Together, these inter-subunit sidechain interactions are important in forming the twisted conformation of the two interacting inter-subunit strands $\beta 2$ and $\beta 18$. However, most of inter-subunit sidechain interactions are lost in mHsp60 (Fig. 4B). The absence of T35 interactions with A516 and T515 (Table 3) releases the restrains on T35 and A516 from forming twisted N-termini of $\beta 2$ and $\beta 18$. Similarly, the much-reduced R34-E517 interactions (4.0/4.7 $\AA$ ) and the absence of R34-V112 interaction release the restrain on E517 from a tight bent of $\beta 18$. Thus, the three mHsp60 unique residues, T35, A516 and V112, diminish the restrains required to form the twisted conformations found in GroEL $\beta 2$ and $\beta 18$, leading to shortened $\beta 2$ and $\beta 18$ in mHsp60. Importantly, the three residues are conserved in higher eukaryotic chaperonins (Fig. 3c).

At the inner side of the inter-subunit $\beta$-sheet, the GroEL K4 sits snugly in the center of a negative cluster. K4 interactions with both E59 and E61 from the neighboring subunit, albeit modest, position K4 to form an effective interaction with D523 (3.6 ̊). The K4-D523 sidechain interaction brings the backbones of K4 and D523 close to form hydrogen bonding, adding twisted ends to $\beta 1$ and $\beta 18$, respectively (Fig. $4 \mathrm{C}$ ). In comparison, with the absence of mHsp60 K2-E522 sidechain interaction, likely affected by the different K2-S57 and K2-D59 interactions when compared with those of K4-E59 and K4-E61 (Table 3), the backbones of K2 and E522 are too far to engage in hydrogen bonding, excluding both residues from $\beta 1$ and $\beta 18$, respectively (Fig. $4 \mathrm{D})$. Thus, the three mHsp60 residues, S57, D59 and E522, contribute to the shortened $\beta 1$ and $\beta 18$. Importantly, the three residues are conserved in higher eukaryotic chaperonins (Fig. 3c), as found with T35, A516 and V112 (above).

Unfolding free energy. The above structural analysis indicates a less stable mHsp60 heptamer than a GroEL heptamer. Consistently, we found that the unfolding free energy $\left(\Delta \mathrm{G}_{\text {unfolding }}\right)$ of $\mathrm{mHsp} 60$ is lower than GroEL $^{\text {SR }}$ (Table 4, Fig. 3d). Our structural analysis also shows the importance of the regions including the N-terminus in the inter-subunit interactions. D3G mutation, associated with MitCHAP60 disease ${ }^{32}$, has been found to destabilize the mHsp60 heptamer ${ }^{33}$. D3 in mHsp60 is the first residue in the already minimal three-residue $\beta 1$. Since Gly has low propensity to form a $\beta$ strand, D3G mutation most likely demolishes $\beta 1$, downgrading the four-stranded to a three-stranded, $\beta 18-\beta 2-\beta 3$, $\beta$-sheet. Consistently, we found that $\Delta \mathrm{G}_{\text {unfolding }}$ of $\mathrm{mHsp} 60^{\mathrm{D} 3 \mathrm{G}}$ was much smaller than the wildtype mHsp60 (Table 4, Fig. 3d).

Enhanced subunit interactions in $\mathrm{mHsp} 60$ heptamer in response to $\mathrm{mHsp} 10$ binding. The mHsp60 subunit interactions are enhanced upon mHsp10 binding. We compared our structure of single-ring

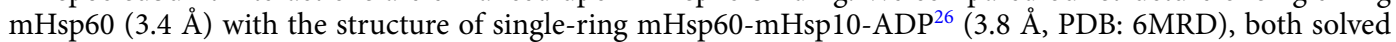
via cryo-EM (Fig. 5a,b). We first focused on the inter-subunit interface in the equatorial domains, since the domains provide the major inter-subunit interactions (above). Strikingly, mHsp10 binding induces significant structural changes in the equatorial domain, promoting the subunit association. The shortened the inter-subunit $\beta 1-\beta 18-\beta 2-\beta 3$ sheet is largely restored to that found in GroEL: $\beta 1$ restores from three to five residues, while $\beta 18$ increases from three to five resides (Fig. S4). Moreover, the inter-subunit $\beta$ sheet serves as a pivoting point to tilt the neighboring equatorial domains towards each other (Fig. $5 c, d)$. As a result, the interface formed via the equatorial domains increases significantly by $29 \%$ from $938 \AA^{2}$ in the mHsp60 alone structure to $1211 \AA^{2}$ in the $\mathrm{mHsp} 60-\mathrm{mHsp} 10$ structure. In addition, both the apical and the intermediate domains undergo large domain movements (below), increasing their contribution to the inter-subunit interaction from 25 to $35 \%$. Overall, the inter-subunit interface of the mHsp60 heptamer increases drastically by $\sim 49 \%$ from $1258 \AA^{2}$ in the mHsp60 alone structure to $1871 \AA^{2}$ in the mHsp60-mHsp10 structure. The mHsp10-induced drastic increase in intersubunit interface in mHsp60 heptamer is consistent with the observation that the presence of $\mathrm{mHsp} 10$ (and ATP) prevents mHsp60 from dissociation ${ }^{20}$. 
a)

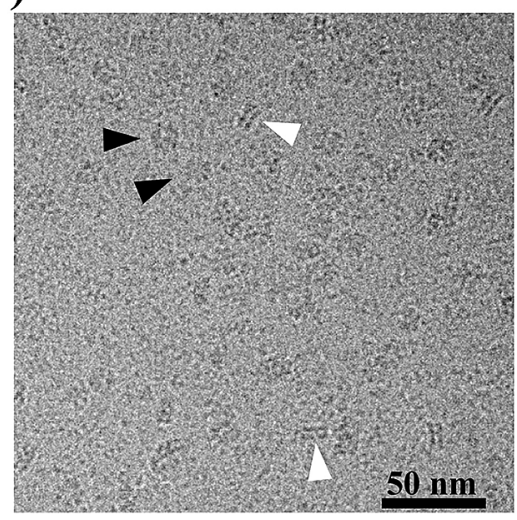

b)

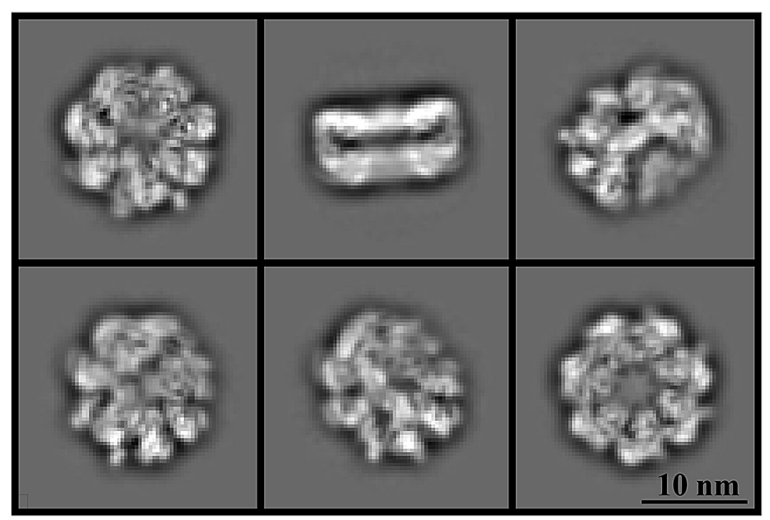

c)
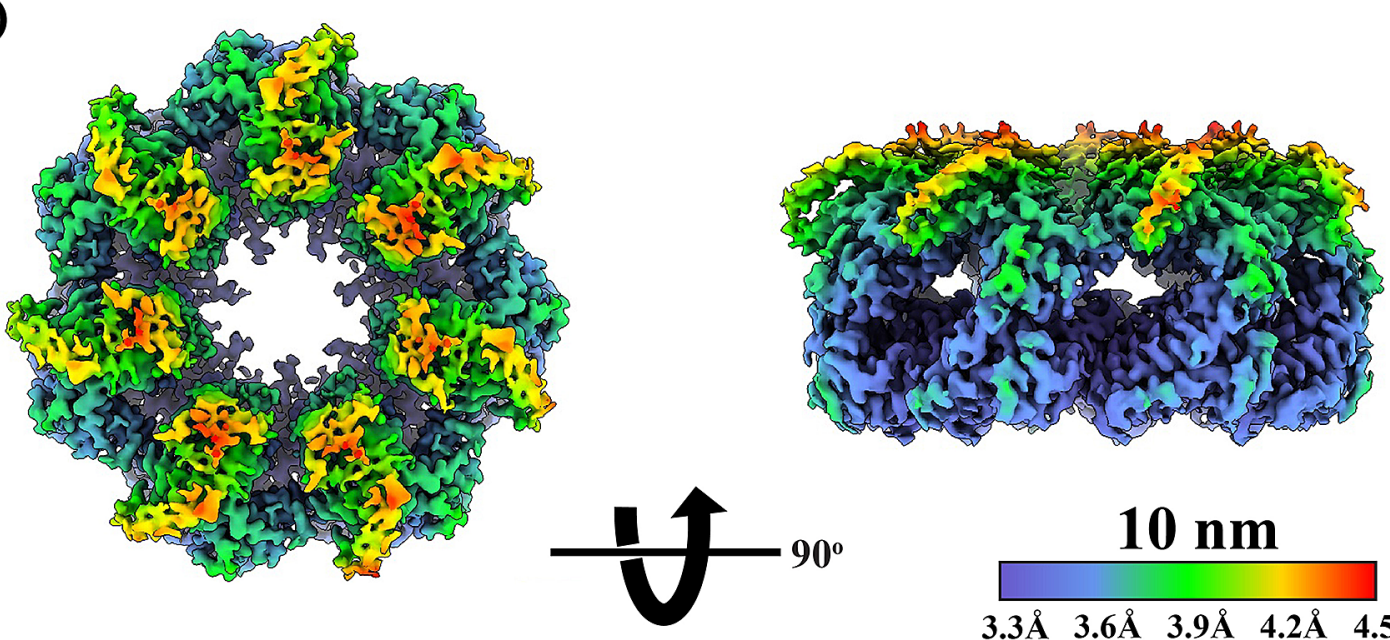

$10 \mathrm{~nm}$

$3.3 \AA 3.6 \AA 3.9 \AA \quad 4.2 \AA \quad 4.5 \AA$

d)

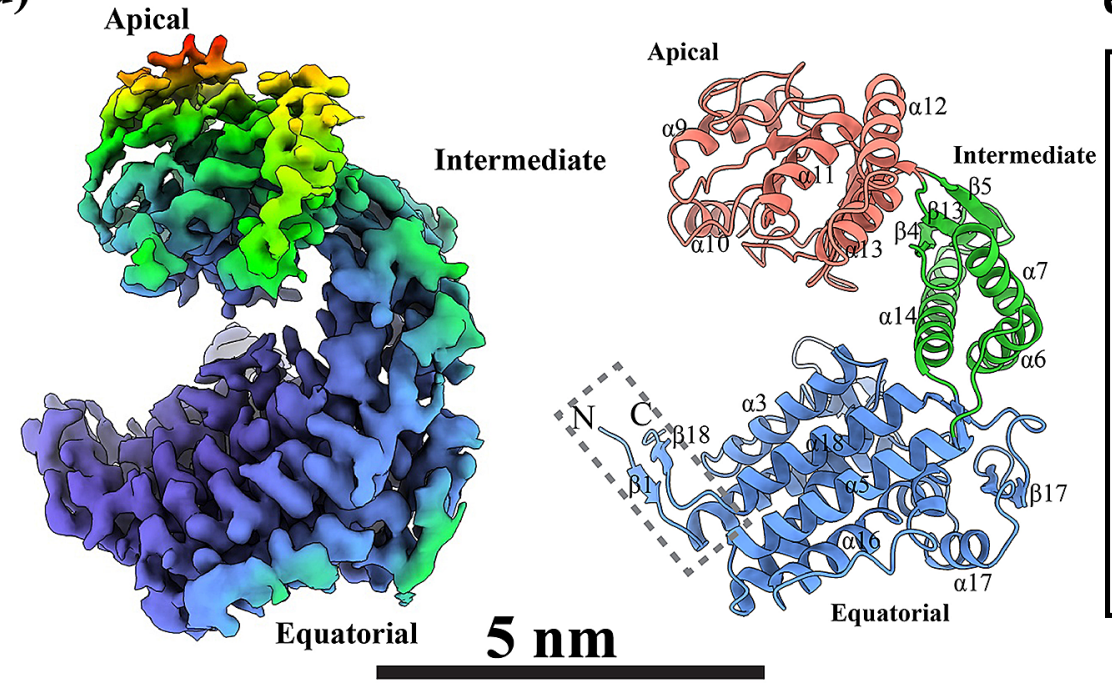

e)

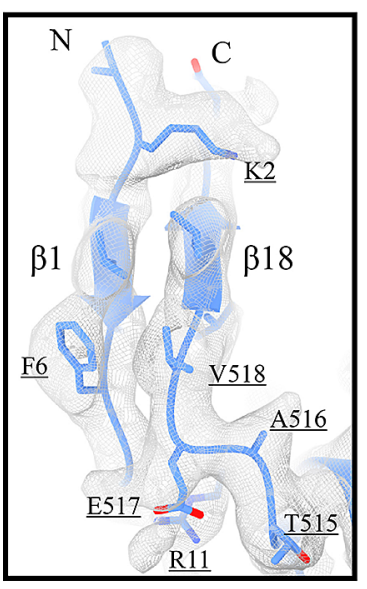

Figure 2. Cryo-EM analysis of mHsp60. (a) A representative cryo-EM micrograph. Side and tilt views are highlighted by white and black arrowheads. (b) Representative reference-free 2D class averages images. (c) Cryo-EM reconstruction of the mHsp60 heptamer colored by local resolution. (d) A mHsp60 subunit displayed in cryo-EM reconstruction with local resolution and in ribbon representation. Ribbon color scheme, blue, green and red for the equatorial, intermediate and apical domains, is the same as Fig. 3C. (e) The EM density at the region involved in the inter-subunit interaction. 


\begin{tabular}{|c|c|c|}
\hline Reconstruction data & mHsp60 (PSU COM) & mHsp60 (NCCAT) \\
\hline \multicolumn{3}{|l|}{ Data collection information } \\
\hline Electron microscope & TFS Titan Krios & TFS Titan Krios \\
\hline Operation voltage $(\mathrm{kV})$ & 300 & 300 \\
\hline Electron detector & Gatan K3 & Gatan K3 \\
\hline Energy filter (slit width in eV) & 30 & no \\
\hline Data collection software & TFS EPU & Leginon \\
\hline Data collection mode & counting & Super-resolution counting \\
\hline Nominal magnification & $120,000 \times$ & $81,000 \times$ \\
\hline Pixel size in $\AA$ (super resolution) & 0.84 & $1.069(0.535)$ \\
\hline Total accumulated dose $\left(\mathrm{e}^{-} / \AA^{2}\right)$ & 44 & $50-57$ \\
\hline \multicolumn{3}{|l|}{ Data process statistics } \\
\hline Box size (pixel) & 320 & 240 \\
\hline Number of particles & 196,060 & 182,600 \\
\hline CTF estimation & Gctf & CTFFIND4 \\
\hline Data processing software & RELION 3.1 & RELION 3.1 \\
\hline Symmetry imposition & C7 & C7 \\
\hline B-factor applied $\left(\AA^{2}\right)$ & -151 & -191 \\
\hline Final resolution $(\AA)$ & 3.5 & 3.5 \\
\hline Combined B-factor $\left(\AA^{2}\right)$ & -180 & \\
\hline Combined resolution $(\AA)$ & 3.4 & \\
\hline EMDB code & EMD-23217 & \\
\hline \multicolumn{3}{|l|}{ Model refinement statistics } \\
\hline $\mathrm{CC}_{\text {masked }}$ (Model vs Data) & 0.73 & \\
\hline $\mathrm{CC}_{\text {volume }}$ (Model vs Data) & 0.71 & \\
\hline RMSD bond length $(\AA)$ & 0.007 & \\
\hline RMSD bond angles $\left({ }^{\circ}\right)$ & 0.699 & \\
\hline \multicolumn{3}{|l|}{ Ramachandran plot } \\
\hline Favored (\%) & 88.85 & \\
\hline Allowed (\%) & \begin{tabular}{|l|}
10.77 \\
\end{tabular} & \\
\hline Outliers (\%) & 0.38 & \\
\hline Clash score & 12.51 & \\
\hline PDB code & 7L7S & \\
\hline
\end{tabular}

Table 2. Cryo-EM data collection, refinement and validation statistics.

Similarly as seen in GroEL upon GroES binding ${ }^{34}$, both the apical and the intermediate domains of mHsp60 undergo large domain movements upon mHsp10 binding (Fig. 5a,b). Specifically, $\alpha 14$ of the intermediate domain rotates downwards to the equatorial domain in $\mathrm{mHsp} 60-\mathrm{mHsp} 10$, closing the nucleotide binding site and locking the bound nucleotide. The three stranded $\beta 4-\beta 13-\beta 5$ sheet in apo mHsp60 is tilted upward and reduced to a two stranded $\beta 4-\beta 13$ sheet in $\mathrm{mHsp} 60-\mathrm{mHsp} 10$. The connected three-stranded $\beta 6-\beta 12-\beta 11$ sheet in mHsp60 is swung $\sim 150^{\circ}$ and increased to a four-stranded $\beta 5-\beta 6-\beta 12-\beta 11$ sheet in mHsp60-mHsp $10 . \alpha 9$ and $\alpha 10$, the main binding site for the substrate protein $\mathrm{s}^{35,36}$, originally facing the chaperonin cavity in $\mathrm{mHsp} 60$, are repositioned upward to interact with mHsp10 in mHsp60-mHsp10. Importantly, the re-positioned apical and intermediate domains are in close contact with their counterparts in the neighboring subunits. As a result, their combined inter-subunit interface increases by $>100 \%$ from $320 \AA^{2}$ in the mHsp60 alone structure to $660 \AA^{2}$ in the mHsp60mHsp10 structure, contributing to stabilizing the mHsp60 heptamer.

\section{Discussion}

Due to its essential role for mitochondrial function and its important roles in human health, mHsp60 has been proposed as potential biomarkers and drug targets ${ }^{5-8}$. However, detailed molecular and structural investigations on mHsp60 mechanism have been hampered by the difficulties in purifying $\mathrm{mHsp} 60$ due to its intrinsic dynamics. The intrinsic dynamics, although a hurdle to detailed molecular investigations on mHsp60, has been implicated in the unique mechanism of mHsp60. Structures of mHsp60 in complex with mHsp10 have been reported ${ }^{25,26}$, however, these structural studies do not address the structural dynamics of mHsp60, because the mHsp60 subunit association is stabilized in the mHsp60-mHsp10 complex. Here we report the structure of mHsp60 alone, the initial functional state in the $\mathrm{mHsp} 60-\mathrm{mHsp} 10$ chaperone reaction. Our structural analysis provides explanations for the dynamics of the $\mathrm{mHsp} 60$ subunit associations and how the $\mathrm{mHsp} 60$ subunit association is enhanced upon mHsp10 binding.

The dynamics of mHsp60 subunit associations is due to the unique sequences at the inter-subunit interface. Many of the six unique mHsp60 sequences result in diminishing the inter-subunit sidechain interactions as 
a)
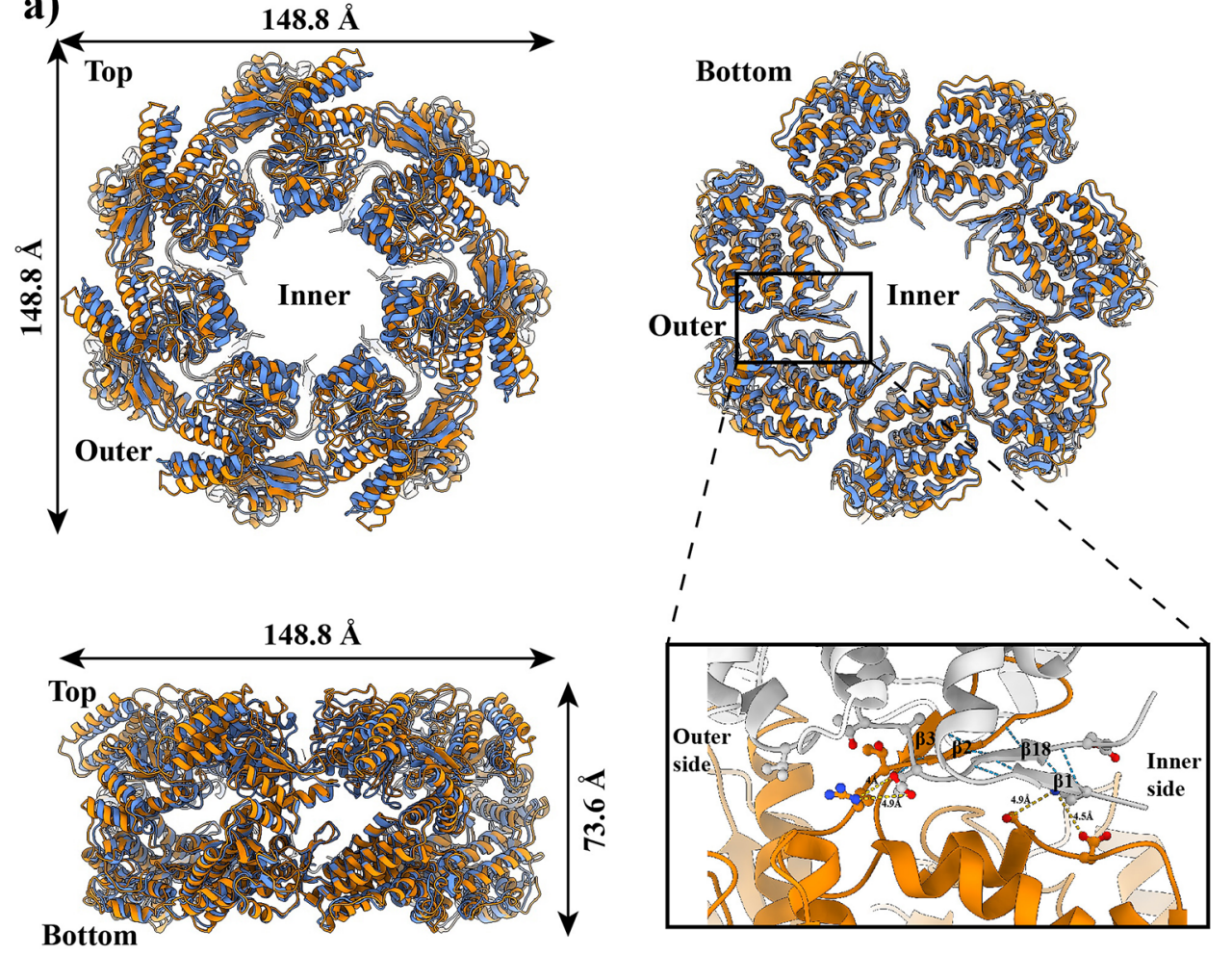

b)
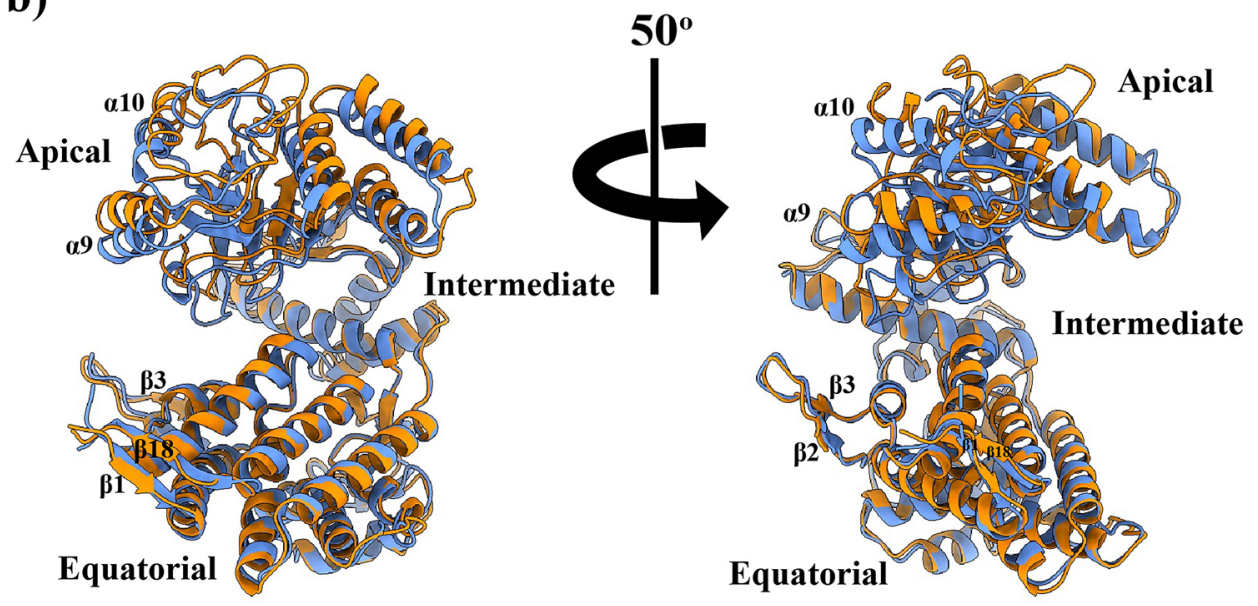

Figure 3. An expanded mHsp60 heptameric ring. (a) Superimpose of mHsp60 (orange) and a GroEL ring (blue; PDB: 5w0s) in top, bottom and side views. A four-stranded $\beta$-sheet, consisting of two strands $\beta 1$ and $\beta 18$ from one subunit (grey) and two strands $\beta 2$ and $\beta 3$ from the other subunit (orange), is located at the center of the cross-ring inter-subunit interface. Residues involved in inter-subunit interactions are highlighted in Fig. 4, and the atomic distances are summarized in Table 3. The mHsp60 heptamer and one GroEL heptamer were superimposed. (b) Overlay of a mHsp60 subunit with a GroEL subunit. The two equatorial domains were superimposed. (c) Sequence alignment among chaperonins from various sources. Secondary structures of mHsp60 and GroEL are based on Chimera: stripped rectangles for $\alpha$ helices and solid rectangles for $\beta$ strands. Color scheme is the same as Fig. 2 d. Residues involved in the inter-subunit interactions that are unique to human or higher eukaryote mouse and bovine (although yeast also has Ser at the corresponding S57 in human) are in red. Four residues forming the conserved inter-ring salt bridges are in dark (K105-E435) and light (R453-E462) shades, in GroEL numbering. For convenience, numbering of GroEL secondary structures is used here. (d) Thermal denaturation of single-ring chaperonins. The molar ellipticity ( $\vartheta$ ) was measured at $222 \mathrm{~nm}$. The solid lines are non-linear regression analysis (Eq. 1). The derived $\Delta \mathrm{H}_{\mathrm{vh}}, \Delta \mathrm{G}_{\text {unfolding }}$ and $\mathrm{T}_{\mathrm{m}}$ values are listed in Table 4. 


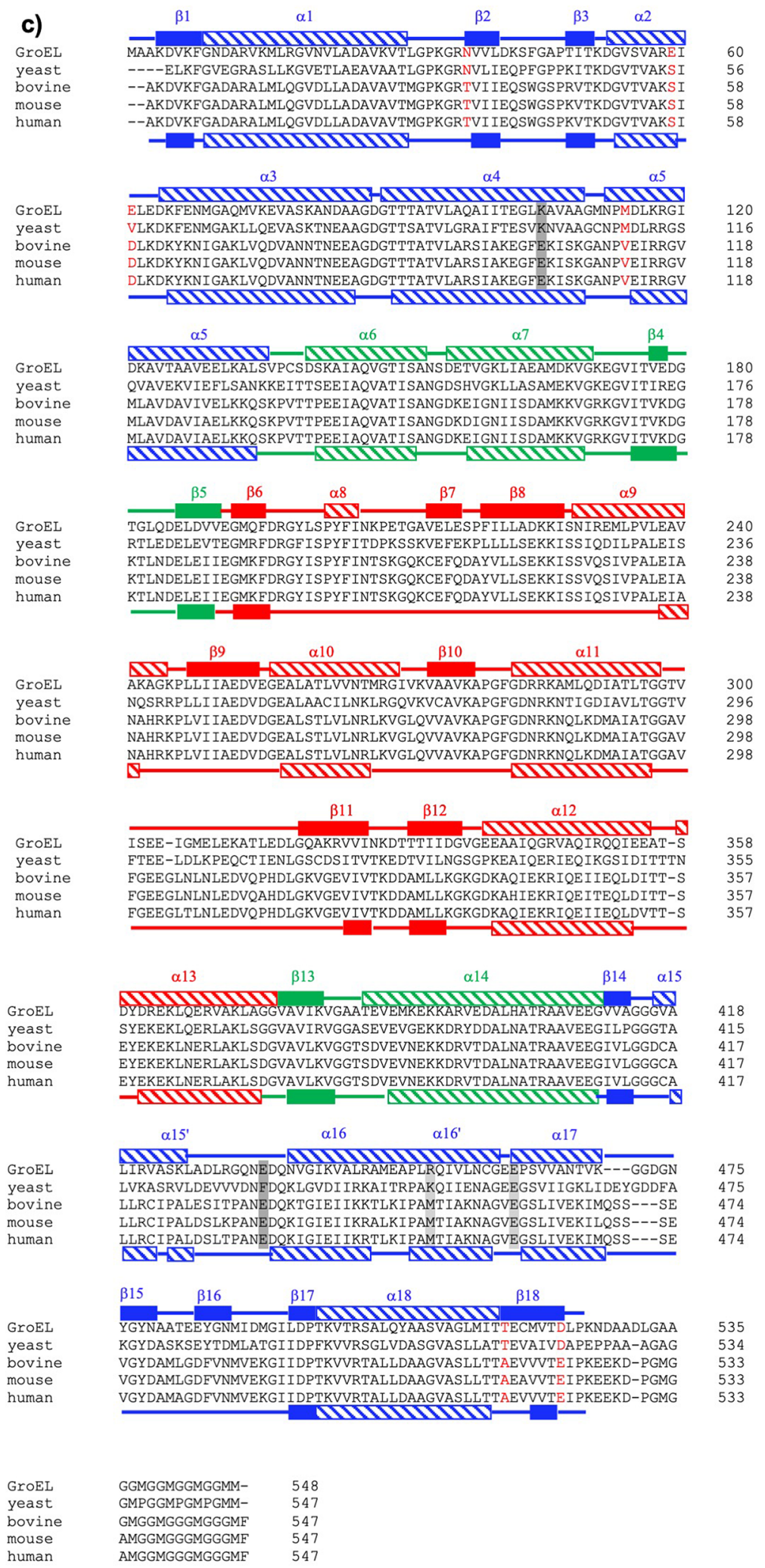

Figure 3. (continued) 


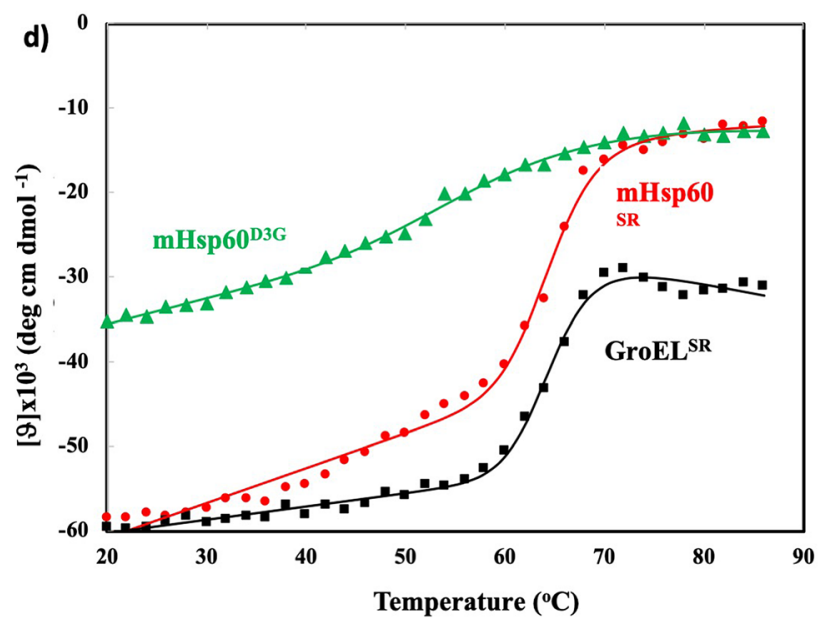

Figure 3. (continued)

\begin{tabular}{|c|c|c|}
\hline Contacts & mHsp60 & GroEL \\
\hline Outer side & $\begin{array}{l}\text { R34 }-\underline{\text { V112 }} \\
\underline{\mathrm{T} 35}=-\underline{\mathrm{A} 516} \\
\underline{\mathrm{T} 515}\end{array}$ & $\begin{array}{r}\text { R36 } \frac{2.8}{\ddots \bullet} \text { E518 } \\
4 .{ }^{\circ} \cdot \underline{M} \text { M114 } \\
\underline{\mathrm{N} 37} \frac{4.5}{2.7} \frac{\mathrm{T} 517}{\mathrm{~T} 516}\end{array}$ \\
\hline Inner side ${ }^{a}$ & 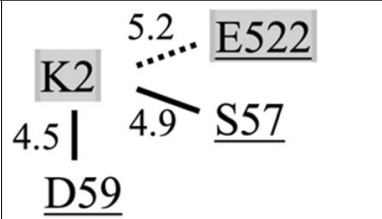 & 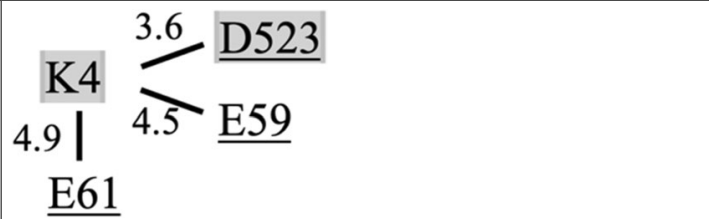 \\
\hline$\beta$-sheet ${ }^{b}$ & 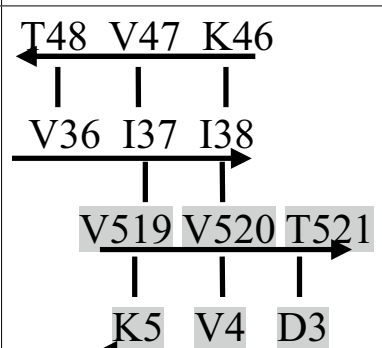 & 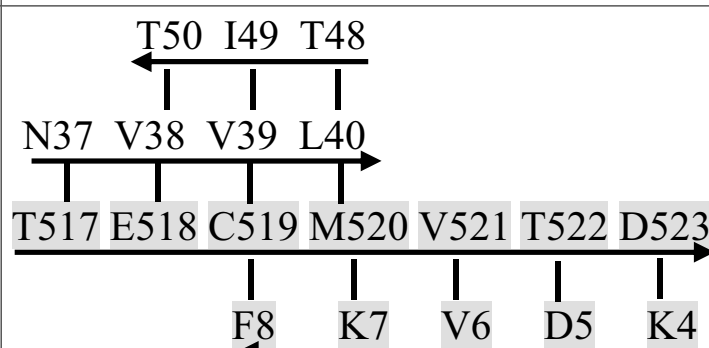 \\
\hline
\end{tabular}

Table 3. Comparison of inter-subunit interactions between $\mathrm{mHsp} 60$ and GroEL. ${ }^{\mathrm{a} C h a r g e-c h a r g e ~ i n t e r a c t i o n s ~}$ between sidechain atoms. Distances shorter than $5 \AA$ in the GroEL structure (cryoEM structure, pdb: $5 \mathrm{w} 0 \mathrm{~s}$ ) and their corresponding values in the $\mathrm{mHsp} 60$ structure are listed. Shade residues are from a neighboring subunit. Residues that are different between mHsp60 and GroEL are underlined. Numbers are distances in angstrom. Dashed lines are missing interactions. The K2-E522 dotted line denotes a distance longer than $5 \AA$, and the R36-M114 dotted line denotes a charge-polar interaction. ${ }^{\mathrm{b}}$ Hydrogen bonds between backbone atoms. Arrows indicate the directions of $\beta$ strands.

found in GroEL. For example, the mHsp60 T35 does not interact with either T515 or A516 of the neighboring subunit, while the corresponding GroEL N37 interacts with T516 and T517 of the neighboring subunit. Moreover, since these unique sequences are located at the ends of $\beta$ strands, losses of their sidechain interactions fail to engage their backbones to conforming to hydrogen bonding, excluding them from $\beta$ strand conformation and shortening the $\beta$ strands. The losses of T35 interactions with T516 and T517 exclude T35 from $\beta 2$ and T516 and T517 from $\beta 18$. The importance of sidechain interactions in extending $\beta$ strands is underscored in GroEL. The N37 interactions with T516 and T517, together with the R36 interaction with E518, restrict the backbones of N37, T517 and E518. As a result, N37 adds a twisted N-terminus to $\beta 2$, and both T517 and E518 extend a bended N-terminal segment to $\beta 18$. The reduced inter-subunit sidechain interactions due to the unique mHsp60 

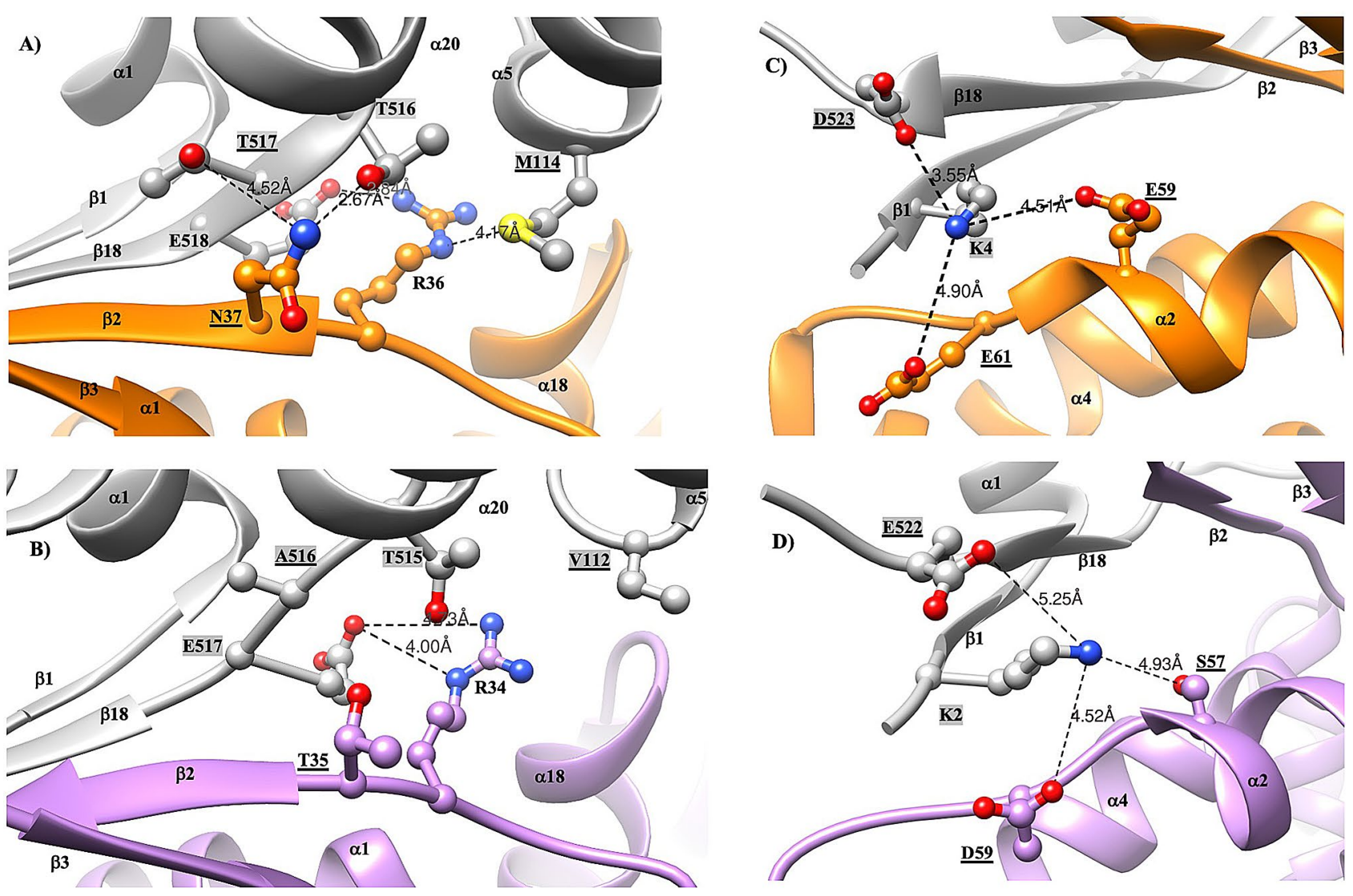

Figure 4. $\mathrm{mHsp} 60$ has reduced inter-subunit interactions compared with GroEL. Charge-charge or chargepolar interactions at the outer side (A, B) and inner side (C, D) of the $\beta$-sheet. $\mathrm{mHsp60}$ subunits are in purple and gray, while GroEL subunits are in orange and gray. Shade residues are from a neighboring subunit; residues that are different between mHsp60 and GroEL are underlined. Numbers, distances in angstrom, are summarized in Table 3.

\begin{tabular}{|l|l|l|l|}
\hline Proteins & $\Delta \mathbf{H}_{\mathbf{v h}}(\mathbf{k c a l} / \mathbf{m o l})^{\mathbf{a}}$ & $\Delta \mathbf{G}_{\text {unfolding }}(\mathbf{k c a l} / \mathbf{m o l})^{\mathbf{b}}$ & $\mathbf{T}_{\mathbf{m}}{ }^{{ }^{\circ}}\left({ }^{\circ} \mathbf{C}\right)$ \\
\hline mHsp60 & 13.92 & 9.59 & 64.7 \\
\hline GroEL $^{\text {SR }}$ & 15.15 & 10.44 & 64.7 \\
\hline mHsp60 $^{\text {DG }}$ & 3.49 & 2.36 & 62.8 \\
\hline
\end{tabular}

Table 4. Thermodynamic parameters from thermal unfolding of $\mathrm{mHsp} 60$ and $\mathrm{GroEL}^{\mathrm{SR}}$ proteins via circular dichoism. ${ }^{\mathrm{a}}$ van't Hoff enthalpy, derived from fitting data to Eq. $1 .{ }^{\mathrm{b}}$ The free energy of unfolding at $20^{\circ} \mathrm{C}$, derived from Eq. 2 . $^{\mathrm{c}}$ The temperature of the transition midpoint, derived from fitting data to Eq. 1 .

sequences, together with the resulting shortened inter-subunit $\beta$-sheets, decrease the inter-subunit interface of mHsp60, accounting for the structural dynamics of $\mathrm{mHsp} 60$ subunit association. Importantly, the unique sequences, T35, S57, D59, V112, A516, and E522, are specific to and conserved within the mitochondrial chaperonins of higher eukaryotes including human. For the lower eukaryotic organisms such as yeast, the sequences at these positions resemble those of GroEL. Moreover, yeast contain sequences for one of the two conserved inter-ring salt bridges, R452-E462 in GroEL, while the higher eukaryotes lack sequences for both salt bridges (Fig. 3c). Thus, the destabilizing sequences are unique to the single ring eukaryotic chaperonins, underscoring their importance in structural dynamics and functional mechanism of the single-ring chaperonins in higher eukaryotic mitochondria.

Binding of cochaperonins induces large structural changes in chaperonins in a nucleotide dependent manner. The large structural changes include an upward rotation of $\alpha 14$ in the equatorial domain, an upward tilt of the $\beta 5 / \beta 13 / \beta 4$ sheet in the intermediate domain, and a large swing of the $\beta 6 / \beta 12 / \beta 11$ sheet and a reorientation of $\alpha 9$ and $\alpha 10$ in the apical domain (Fig. 5a). For GroEL, the large structural changes do not affect the inter-subunit interface: the interface remains largely the same before $\left(1583 \AA^{2}\right)$ and after $\left(1594 \AA^{2}\right)$ GroES binding (PDB: 1PCQ; the trans and cis GroEL ring, respectively). In addition, the contributions from individual domains to the inter-subunit interface remain largely the same. For example, the equatorial domains contribute $62 \%$ and $64 \%$ of 


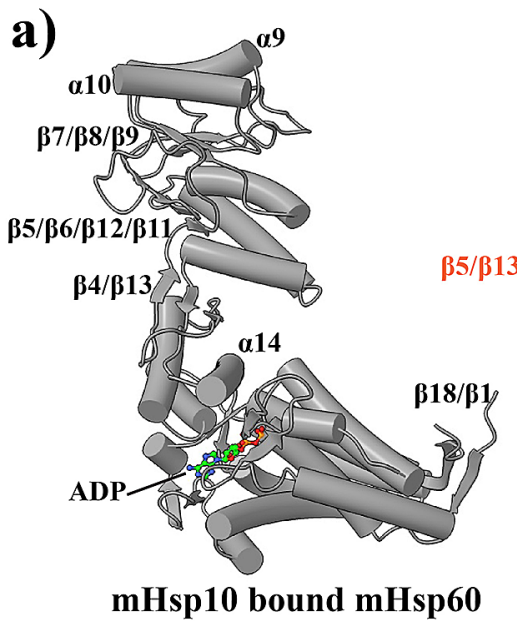

c)

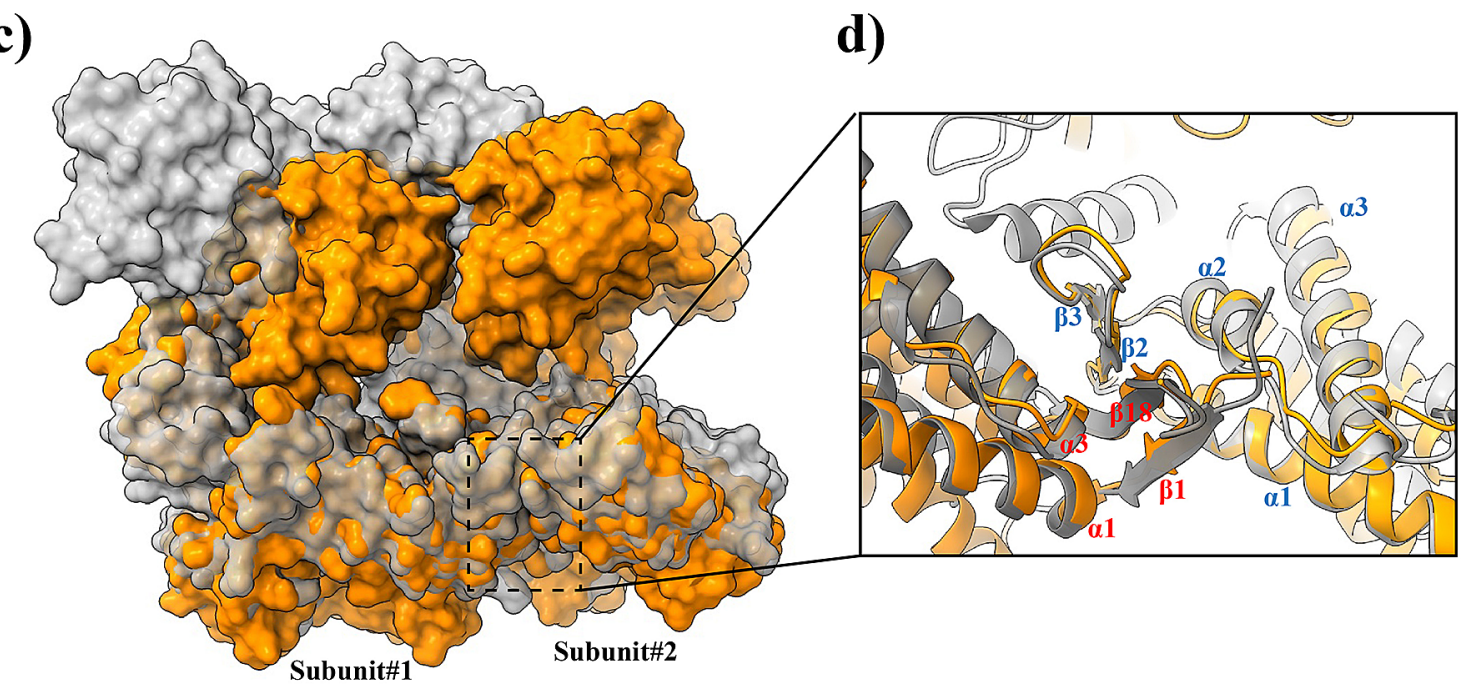

Figure 5. mHsp10 binding stabilizes mHsp60 subunit association. (a) Ribbon diagrams of mHsp60-mHsp10 (PDB: 6MRD, grey) and mHsp60 (orange) subunits. Structural elements mentioned in text are annotated.

(b) Overlay of one $\mathrm{mHsp60-mHsp10} \mathrm{subunit} \mathrm{and} \mathrm{one} \mathrm{mHsp} 60$ subunit in ribbon diagram representation. (c) Overlay of $\mathrm{mHsp} 60$ with $\mathrm{mHsp60-mHsp10} \mathrm{in} \mathrm{surface} \mathrm{representation.} \mathrm{The} \mathrm{mHsp60}$ heptameric ring in mHsp60mHsp10 was superimposed with the apo $\mathrm{mHsp} 60$ heptamer. Only two subunits from each structure are shown. (d) Closeup on the inter-subunit interface around the $\beta 1-\beta 18-\beta 2-\beta 3$ sheet. The sheet acts as a pivotal point to bring the neighboring equatorial domains closer in $\mathrm{mHsp} 60-\mathrm{mHsp} 10$ than in $\mathrm{mHsp60}$. Secondary structures are labeled in blue for one subunit and in red for the other subunit.

the total interface before and after GroES binding, respectively. Moreover, structure of the inter-subunit $\beta 1-\beta 18$ $\beta 2-\beta 3$ sheet remains the same after GroES binding. This structural analysis suggests that the subunit association in the GroEL ring, i.e., the stability of the GroEL ring, is not affected by GroES binding. In a striking contrast, $\mathrm{mHsp} 10$ binding affects inter-subunit interaction in $\mathrm{mHsp} 60$ drastically. Upon $\mathrm{mHsp} 10$ binding, the overall inter-subunit interface in $\mathrm{mHsp} 60$ increases by $49 \%$, and the contributions from the equatorial domains and the apical/intermediate domains increase by $29 \%$ and $105 \%$, respectively. In addition, the shortened inter-subunit $\beta 1-\beta 18-\beta 2-\beta 3$ sheet of $\mathrm{mHsp} 60$ is restored to the same as in GroEL or the GroEL-GroES complex (Fig. S4). Thus, unlike GroEL, $\mathrm{mHsp} 60$ subunit association, i.e., $\mathrm{mHsp60}$ heptameric stability, is enhanced in the presence of bound mHsp10. The mHsp10 effect on $\mathrm{mHsp} 60$ stability implicates the importance of structural dynamics in heptameric mHsp60, underscoring the mechanistic differences between mHsp60-mHsp10 and GroEL-GroES.

\section{Methods}

Protein purification. Gene encoding the mature mHsp60 sequence (without the mitochondrial targeting signal) was synthesized with codons optimized for protein expression in E. coli, and was inserted into pBbE5a with NdeI/BamHI sites. pBbE5a-mHsp60 plasmid was transformed to BL21DE3 for protein expression. Cells were grown in $\mathrm{LB}$ at $37^{\circ} \mathrm{C}$ until $\mathrm{OD}_{600}=0.6$, protein expression was induced with $0.4 \mathrm{mM}$ IPTG, and cells continued to grow for $5 \mathrm{~h}$. Protein purification was modified from a published protocol ${ }^{22}$. Briefly, cells were suspended in Buffer A (10 mM KHEPES, pH 7.4, 1 mM EDTA, $1 \mathrm{mM}$ DDT and 5\% glycerol) and lyzed via sonication. After centrifugation, ammoniate sulfate was added to the clear lysate to a final concentration of $20 \%$. The solution was centrifuged, and the supernatant was loaded onto a butyl column, preequilibrated with $20 \%\left(\mathrm{NH}_{4}\right)_{2} \mathrm{SO}_{4}$ 
ammoniate sulfate in Buffer A. After extensive wash including with $20 \%$ methanol, $20 \%$ ammoniate sulfate in Buffer $\mathrm{A}$, the bound protein was eluted with a linear gradient of $20-0 \%\left(\mathrm{NH}_{4}\right)_{2} \mathrm{SO}_{4}$. The $\mathrm{mHsp} 60$ containing fractions were combined and $\left(\mathrm{NH}_{4}\right)_{2} \mathrm{SO}_{4}$ was added to a final $60 \%$ to precipitate $\mathrm{mHsp} 60$. The precipitated mHsp60 was dissolved in $100 \mathrm{mM} \mathrm{NaCl}$ in Buffer $\mathrm{A}$, and the solution was subjected to gel filtration purification using a Superdex 200 column (GE Healthcare). Fractions containing single ring mHsp60 were concentrated to a desired concentration, and the freshly concentrated mHsp60 was frozen on the EM grids or used within the same day for analysis. $\mathrm{mHsp} 60^{\mathrm{D} 3 \mathrm{G}}$ was generated using QuickChange (Qiagen), and purified as above. GroEL and GroEL ${ }^{\mathrm{SR}}$ were purified as before ${ }^{37}$.

Dynamic light scattering (DLS). Freshly purified mHsp60, GroEL and GroEL ${ }^{\mathrm{SR}}$ were subjected to DLS analysis using a Zetasizer Nano-S dynamic light scattering instrument (Malvern Instruments). Samples were in $100 \mathrm{mM} \mathrm{NaCl}, 10 \mathrm{mM}$ KHepes, $1 \mathrm{mM}$ EDTA, $1 \mathrm{mM}$ DTT and 5\% glycerol, and the temperature was $25^{\circ} \mathrm{C}$. DLS data were collected within few hours of purification and for the next six days. Measurements were repeated more than once for each sample.

ATPase assay. The steady-state ATP hydrolysis rate was measured using the malachite green assay, as described in our previous work ${ }^{37}$. $\mathrm{mHsp60}$, GroEL or GroEL ${ }^{\mathrm{SR}}$ was added to TEA buffer (50 mM triethanolamine, $\mathrm{pH} 7.5,50 \mathrm{mM} \mathrm{KCl}$, and $20 \mathrm{mM} \mathrm{MgCl}$ ) to a final concentration of $0.25 \mathrm{uM}$ (heptameric mHsp60 or $\mathrm{GroEL}^{\mathrm{SR}}$ ) or $0.125 \mathrm{uM}$ (tetradecameric GroEL). The final concentration of mHsp60 or GroES was $0.3 \mathrm{uM}$. The solution was incubated at $25^{\circ} \mathrm{C}$ for $10 \mathrm{~min}$. The hydrolysis was initiated by addition of $100 \mathrm{mM} \mathrm{pH} \mathrm{7.0} \mathrm{ATP} \mathrm{to} \mathrm{a}$ final concentration of $2 \mathrm{mM}$ and followed every $3 \mathrm{~min}$ for $21 \mathrm{~min}$ using the malachite green assay. Measurements were repeated more than once for each sample.

MDH folding assay. Refolded MDH was assayed by measuring its ability to convert NADH to $\mathrm{NAD}^{+}$, as described in our previous work ${ }^{37}$. $\mathrm{MDH}$ was unfolded in TEA buffer including $3 \mathrm{M} \mathrm{GdmHCl}$ to a final concentration of $36.7 \mathrm{uM}$ (monomeric $\mathrm{MDH}$ ). To refold $\mathrm{MDH}, 2.75 \mathrm{ul}$ of unfolded $\mathrm{MDH}$ was diluted at 1:100 (v/v) to a

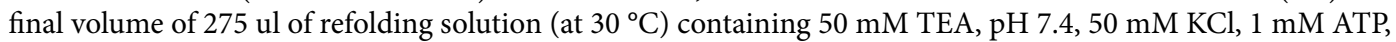
and $2 \mathrm{uM}$ heptameric mHsp60 or GroEL ${ }^{\mathrm{SR}}$ or $2 \mathrm{uM}$ tetradecameric GroEL) with $4 \mathrm{uM}$ or without mHsp10 or GroES. At desired time intervals, $20 \mathrm{ul}$ of reaction solution was removed and mixed with $1 \mathrm{ml}$ of NADH assay solution (50 mM TrisCl, pH 7.4, $10 \mathrm{mM}$ DTT, $0.2 \mathrm{mM} \mathrm{NADH}, 1 \mathrm{mM}$ ketomalonate), and absorption at $340 \mathrm{~nm}$ was taken to monitor the decrease of NADH. As a positive control, activity of $367 \mathrm{nM}$ native $\mathrm{MDH}$ (monomeric concentration) was measured at the same time intervals and was taken as $100 \%$ activity. Measurements were repeated more than once for each sample.

Circular dichoism (CD). Protein stocks were in $100 \mathrm{mM} \mathrm{NaF}, 10 \mathrm{mM}$ KHEPES, pH 7.4, 1 mM EDTA, 1 mM DDT and 5\% glycerol. Proteins were tenfold diluted to $100 \mathrm{mM} \mathrm{NaF}, 10 \mathrm{mM} \mathrm{KHEPES}, \mathrm{pH}$ 7.4, $1 \mathrm{mM}$ EDTA and $1 \mathrm{mM}$ TCEP. Samples were heated from 20 to $86^{\circ} \mathrm{C}$ with a heating rate of $2.0^{\circ} \mathrm{C} / \mathrm{min}$ by a Jasco Peltier thermo control device. The CD signal at $222 \mathrm{~nm}$ was recorded by a J-715 CD spectrometer every $2{ }^{\circ} \mathrm{C}$ with a 4 -s averaging time. Measurements were repeated more than once for each sample. Thermal denaturation was analyzed by fitting the data to a two-state model according to the following equation ${ }^{38}$ :

$$
y=\frac{y_{N}+m_{N} T+\left(y_{D}+m_{D} T\right) \exp \left[\Delta H_{v h} / R\left(1 / T_{m}-1 / T\right)\right]}{1+\exp \left[\Delta H_{v h} / R\left(1 / T_{m}-1 / T\right)\right]}
$$

where $y$ is the observed CD signal at $222 \mathrm{~nm}, y_{\mathrm{N}}$ and $y_{\mathrm{D}}$ are the derived signals for the native and denatured samples, and $m_{\mathrm{N}}$ and $m_{\mathrm{D}}$ are the derived baseline slopes for the native and denatured samples, $\mathrm{T}$ is the temperature, $\Delta \mathrm{H}_{v h}$ is the van' Hoff enthalpy, $\mathrm{R}$ is the gas constant and $\mathrm{T}_{\mathrm{m}}$ is the temperature of the transition midpoint. The free energy of unfolding at specific temperature is estimated from a modified Gibbs-Helmholtz equation, assuming no heat capacity change between the denature and native conformations: $\Delta \mathrm{Cp}=\mathrm{C}_{\mathrm{p}}($ denatured $)-\mathrm{C}_{\mathrm{p}}($ native $)=0$

$$
\Delta G(T)=\Delta H_{v h}\left(1-\frac{T}{T_{m}}\right)-\Delta C_{p}\left[\left(T_{m}-T\right)+T \ln \left(T / T_{m}\right)\right]
$$

Cryo-EM data collection, image analysis, and model building. Protein sample prepared in the same batch were submitted to two facilities PSU COM and NCCAT for cryo-EM data collection. Detailed methods for cryo-EM are described in the supplementary method section. In general, approximate $4 \mu \mathrm{l}$ of $\mathrm{mHsp} 60$ in $\sim 9 \mathrm{mg} / \mathrm{ml}$ were applied to Quantifoil ${ }^{\text {mi }}$ holey carbon grids. Vitrification was performed using Thermo Fisher Scientific (TFS) Vitrobot Mark IV under 100\% humidity. Frozen-hydrated specimens were loaded into Thermo Fisher Scientific Titan Krios operated at $300 \mathrm{kV}$ equipped with Gatan K3 camera. Data collection was done by using TFS EPU or Leginon ${ }^{39}$ with (at $30 \mathrm{eV}$ ) or without energy filter. Initial data quality assessment was done by RELION and Appion ${ }^{40}$. Dose weighted motion corrected was done by MotionCor $2^{41}$ and CTF estimation was done by ctffind $4^{42}$ and $\mathrm{Gctf}^{43}$. Particles were semi-manually picked by e2boxer.py in EMAN $2^{44}$. Reference-free 2D classification, initial model building, 3D classification, 3D refinement, and CTF refinement were performed in RELION ${ }^{45}$. A total number of 182,600 particles were used to build the final $3 \mathrm{D}$ reconstruction at a resolution of $3.5 \AA$ for NCCAT data and a total number of 196,060 particles were used in the final 3D reconstructions at a resolution of $3.5 \AA$ for PSU COM data (Table 2). To combined final 3D models, the unfiltered odd and even electron density maps from both datasets were scaled based on their size and density and summed in the real 
space. The combined 3D model gave an estimated resolution at 3.4 $\AA$. Cryo-EM 3D density map was rendered by UCSF ChimeraX ${ }^{46}$. Atomic model for $\mathrm{mHsp} 60$ was built using GroEL coordinate (PDB 1PCQ) as a template. Mutation and real space refinement were performed in $\operatorname{Coot}^{47}$ and Phenix ${ }^{48}$.

Received: 27 May 2021; Accepted: 5 July 2021

Published online: 20 July 2021

\section{References}

1. Cheng, M. Y. et al. Mitochondrial heat-shock protein hsp60 is essential for assembly of proteins imported into yeast mitochondria. Nature 337, 620-625. https://doi.org/10.1038/337620a0 (1989).

2. Prasad, T. K., Hack, E. \& Hallberg, R. L. Function of the maize mitochondrial chaperonin hsp60: Specific association between hsp60 and newly synthesized F1-ATPase alpha subunits. Mol. Cell Biol. 10, 3979-3986. https://doi.org/10.1128/mcb.10.8.3979 (1990).

3. Koll, H. et al. Antifolding activity of hsp60 couples protein import into the mitochondrial matrix with export to the intermembrane space. Cell 68, 1163-1175. https://doi.org/10.1016/0092-8674(92)90086-r (1992).

4. Ostermann, J., Horwich, A. L., Neupert, W. \& Hartl, F. U. Protein folding in mitochondria requires complex formation with hsp60 and ATP hydrolysis. Nature 341, 125-130. https://doi.org/10.1038/341125a0 (1989).

5. Nakamura, H. \& Minegishi, H. HSP60 as a drug target. Curr. Pharm. Des. 19, 441-451 (2013).

6. Pace, A. et al. Hsp60, a novel target for antitumor therapy: Structure-function features and prospective drugs design. Curr. Pharm. Des. 19, 2757-2764. https://doi.org/10.2174/1381612811319150011 (2013).

7. Cappello, F. et al. Hsp60 chaperonopathies and chaperonotherapy: Targets and agents. Expert Opin. Ther. Targets 18, 185-208. https://doi.org/10.1517/14728222.2014.856417 (2014).

8. Meng, Q., Li, B. X. \& Xiao, X. Toward developing chemical modulators of Hsp60 as potential therapeutics. Front. Mol. Biosci. 5, 35. https://doi.org/10.3389/fmolb.2018.00035 (2018)

9. Gupta, R. S. in The Chaperonins (ed R. Ellis) Ch. 2, 27-57 (Academic Press, 1996).

10. Sigler, P. B. et al. Structure and function in GroEL-mediated protein folding. Annu. Rev. Biochem. 67, 581-608 (1998).

11. Thirumalai, D. \& Lorimer, G. H. Chaperonin-mediated protein folding. Annu. Rev. Biophys. Biomol. Struct. 30, 245-269 (2001).

12. Hartl, F. U. \& Hayer-Hartl, M. Molecular chaperones in the cytosol: From nascent chain to folded protein. Science 295, 1852-1858 (2002).

13. Horwich, A. L., Farr, G. W. \& Fenton, W. A. GroEL-GroES-mediated protein folding. Chem. Rev. 106, 1917-1930 (2006).

14. Lin, Z. \& Rye, H. S. GroEL-mediated protein folding: Making the impossible, possible. Crit. Rev. Biochem. Mol. Biol. 41, 211-239 (2006).

15. Weber, F., Keppel, F., Georgopoulos, C., Hayer-Hartl, M. K. \& Hartl, F. U. The oligomeric structure of GroEL/GroES is required for biologically significant chaperonin function in protein folding. Nat. Struct. Biol. 5, 977-985. https://doi.org/10.1038/2952 (1998).

16. Weissman, J. S. et al. Mechanism of GroEL action: Productive release of polypeptide from a sequestered position under GroES. Cell 83, 577-587 (1995).

17. Viitanen, P. V. et al. Mammalian mitochondrial chaperonin 60 functions as a single toroidal ring. J. Biol. Chem. 267, 695-698 (1992).

18. Brocchieri, L. \& Karlin, S. Conservation among HSP60 sequences in relation to structure, function, and evolution. Protein Sci. 9, 476-486. https://doi.org/10.1110/ps.9.3.476 (2000).

19. Nielsen, K. L. \& Cowan, N. J. A single ring is sufficient for productive chaperonin-mediated folding in vivo. Mol. Cell 2, 93-99 (1998).

20. Levy-Rimler, G. et al. The effect of nucleotides and mitochondrial chaperonin 10 on the structure and chaperone activity of mitochondrial chaperonin 60. Eur. J. Biochem. 268, 3465-3472 (2001).

21. Enriquez, A. S. et al. The human mitochondrial Hsp60 in the APO conformation forms a stable tetradecameric complex. Cell Cycle 16, 1309-1319. https://doi.org/10.1080/15384101.2017.1321180 (2017).

22. Ishida, R. et al. Physicochemical properties of the mammalian molecular chaperone HSP60. Int. J. Mol. Sci. https://doi.org/10. 3390/ijms19020489 (2018).

23. Vilasi, S. et al. Chaperonin of group I: Oligomeric spectrum and biochemical and biological implications. Front. Mol. Biosci. 4, 99. https://doi.org/10.3389/fmolb.2017.00099 (2017).

24. Parnas, A. et al. Identification of elements that dictate the specificity of mitochondrial Hsp60 for its co-chaperonin. PLoS ONE 7, e50318. https://doi.org/10.1371/journal.pone.0050318 (2012).

25. Nisemblat, S., Yaniv, O., Parnas, A., Frolow, F. \& Azem, A. Crystal structure of the human mitochondrial chaperonin symmetrical football complex. Proc. Natl. Acad. Sci. U. S. A. 112, 6044-6049. https://doi.org/10.1073/pnas.1411718112 (2015).

26. Gomez-Llorente, Y. et al. Structural basis for active single and double ring complexes in human mitochondrial Hsp60-Hsp10 chaperonin. Nat. Commun. 11, 1916. https://doi.org/10.1038/s41467-020-15698-8 (2020).

27. Viitanen, P. V. et al. Purification of mammalian mitochondrial chaperonin 60 through in vitro reconstitution of active oligomers. Methods Enzymol. 290, 203-217 (1998).

28. Klebl, D. P. et al. Cryo-EM structure of human mitochondrial HSPD1. iScience 24, 102022. https://doi.org/10.1016/j.isci.2020. 102022 (2021).

29. Okamoto, T. et al. Functional structure and physiological functions of mammalian wild-type HSP60. Arch. Biochem. Biophys. 586, 10-19. https://doi.org/10.1016/j.abb.2015.09.022 (2015).

30. Braig, K., Adams, P. D. \& Brunger, A. T. Conformational variability in the refined structure of the chaperonin GroEL at 2.8 A resolution. Nat. Struct. Biol. 2, 1083-1094. https://doi.org/10.1038/nsb1295-1083 (1995).

31. Chaudhry, C. et al. Role of the gamma-phosphate of ATP in triggering protein folding by GroEL-GroES: function, structure and energetics. EMBO J. 22, 4877-4887. https://doi.org/10.1093/emboj/cdg477 (2003).

32. Magen, D. et al. Mitochondrial hsp60 chaperonopathy causes an autosomal-recessive neurodegenerative disorder linked to brain hypomyelination and leukodystrophy. Am. J. Hum. Genet. 83, 30-42. https://doi.org/10.1016/j.ajhg.2008.05.016 (2008).

33. Parnas, A. et al. The MitCHAP-60 disease is due to entropic destabilization of the human mitochondrial Hsp60 oligomer. J. Biol. Chem. 284, 28198-28203. https://doi.org/10.1074/jbc.M109.031997 (2009).

34. Xu, Z., Horwich, A. L. \& Sigler, P. B. The crystal structure of the asymmetric GroEL-GroES-(ADP)7 chaperonin complex. Nature 388, 741-750 (1997).

35. Fenton, W. A., Kashi, Y., Furtak, K. \& Horwich, A. L. Residues in chaperonin GroEL required for polypeptide binding and release. Nature 371, 614-619. https://doi.org/10.1038/371614a0 (1994).

36. Chen, L. \& Sigler, P. B. The crystal structure of a GroEL/peptide complex: Plasticity as a basis for substrate diversity. Cell 99 , 757-768. https://doi.org/10.1016/s0092-8674(00)81673-6 (1999). 
37. Illingworth, M., Ramsey, A., Zheng, Z. \& Chen, L. Stimulating the substrate folding activity of a single ring GroEL variant by modulating the cochaperonin GroES. J. Biol. Chem. 286, 30401-30408. https://doi.org/10.1074/jbc.M111.255935 (2011).

38. Santoro, M. M. \& Bolen, D. W. Unfolding free energy changes determined by the linear extrapolation method. 1. Unfolding of phenylmethanesulfonyl alpha-chymotrypsin using different denaturants. Biochemistry 27, 8063-8068. https://doi.org/10.1021/ bi00421a014 (1988).

39. Suloway, C. et al. Automated molecular microscopy: The new Leginon system. J. Struct. Biol. 151, 41-60. https://doi.org/10.1016/j. jsb.2005.03.010 (2005).

40. Lander, G. C. et al. Appion: An integrated, database-driven pipeline to facilitate EM image processing. J. Struct. Biol. 166, 95-102. https://doi.org/10.1016/j.jsb.2009.01.002 (2009).

41. Zheng, S. Q. et al. MotionCor2: Anisotropic correction of beam-induced motion for improved cryo-electron microscopy. Nat. Methods 14, 331-332. https://doi.org/10.1038/nmeth.4193 (2017).

42. Rohou, A. \& Grigorieff, N. CTFFIND4: Fast and accurate defocus estimation from electron micrographs. J. Struct. Biol. 192, 216-221. https://doi.org/10.1016/j.jsb.2015.08.008 (2015)

43. Zhang, K. Gctf: Real-time CTF determination and correction. J. Struct. Biol. 193, 1-12. https://doi.org/10.1016/j.jsb.2015.11.003 (2016).

44. Tang, G. et al. EMAN2: An extensible image processing suite for electron microscopy. J. Struct. Biol. 157, 38-46. https://doi.org/ 10.1016/j.jsb.2006.05.009 (2007).

45. Scheres, S. H. RELION: Implementation of a Bayesian approach to cryo-EM structure determination. J. Struct. Biol. 180, 519-530. https://doi.org/10.1016/j.jsb.2012.09.006 (2012).

46. Goddard, T. D. et al. UCSF ChimeraX: Meeting modern challenges in visualization and analysis. Protein Sci. 27, 14-25. https:// doi.org/10.1002/pro.3235 (2018).

47. Emsley, P. \& Cowtan, K. Coot: Model-building tools for molecular graphics. Acta Crystallogr. D Biol. Crystallogr. 60, $2126-2132$. https://doi.org/10.1107/S0907444904019158 (2004).

48. Adams, P. D. et al. PHENIX: A comprehensive Python-based system for macromolecular structure solution. Acta Crystallogr. D Biol. Crystallogr. 66, 213-221. https://doi.org/10.1107/S0907444909052925 (2010).

\section{Acknowledgements}

We gratefully acknowledge the Pennsylvania State University College of Medicine for access to the Cryo-EM (RRID: SCR_021178) and the HPC core facilities. J.W. was supported by the startup fund from Penn State University College of Medicine. We thank Edward Eng, Christina Zimanyi and Eugene Chua of the National Center for CryoEM Access and Training (NCCAT) at the New York Structural Biology Center (NYSBC). Some of this work was performed at NCCAT and the Simons Electron Microscopy Center located at NYSBC, supported by the NIH Common Fund Transformative High Resolution Cryo-Electron Microscopy program (U24 GM129539) and by Grants from the Simons Foundation (SF349247) and NY State Assembly Majority.

\section{Author contributions}

J.W. performed the cryo-EM studies. L.C. performed molecular biology, protein purification and characterization, CD and structural analysis. J.W. and L.C. wrote the paper. L.C. designed the research.

\section{Competing interests}

The authors declare no competing interests.

\section{Additional information}

Supplementary Information The online version contains supplementary material available at https://doi.org/ 10.1038/s41598-021-94236-y.

Correspondence and requests for materials should be addressed to J.C.-Y.W. or L.C.

Reprints and permissions information is available at www.nature.com/reprints.

Publisher's note Springer Nature remains neutral with regard to jurisdictional claims in published maps and institutional affiliations.

(c) Open Access This article is licensed under a Creative Commons Attribution 4.0 International License, which permits use, sharing, adaptation, distribution and reproduction in any medium or format, as long as you give appropriate credit to the original author(s) and the source, provide a link to the Creative Commons licence, and indicate if changes were made. The images or other third party material in this article are included in the article's Creative Commons licence, unless indicated otherwise in a credit line to the material. If material is not included in the article's Creative Commons licence and your intended use is not permitted by statutory regulation or exceeds the permitted use, you will need to obtain permission directly from the copyright holder. To view a copy of this licence, visit http://creativecommons.org/licenses/by/4.0/.

(C) The Author(s) 2021, corrected publication 2021 